\title{
CORRELATION BETWEEN INTESTINAL MICROBIOTA AND GROWTH OF WHITE SHRIMP (LITOPENAEUS VANNAMEI)
}

\author{
WU, J. Y. $.^{1,2}-$ YAN, M. C. ${ }^{1,2}-$ SANG, Y. ${ }^{1,2}-$ LI, F. ${ }^{1,2}-$ LUO, K. ${ }^{1,2}-$ HU, L. H. ${ }^{1,2^{*}}$ \\ ${ }^{I}$ Zhejiang Key Lab of Exploitation and Preservation of Coastal Bio-Resource, Zhejiang \\ Mariculture Research Institute, Wenzhou 325005, China \\ ${ }^{2}$ Wenzhou Key Laboratory of Marine Biological Genetics and Breeding, Zhejiang Mariculture \\ Research Institute, Wenzhou 325005, China \\ Corresponding author \\ e-mail: 51275316@qq.com; phone: +86-138-6876-1896
}

(Received $15^{\text {th }}$ Jun 2021; accepted $3^{\text {rd }}$ Sep 2021)

\begin{abstract}
Although intestinal microbiota is closely related to the growth of the host, it is still unclear how it affects the growth of white shrimp. To elucidate the potential processes of intestinal microbiota affecting white shrimp growth, in this study, we compared the intestinal microbiota compositions and metabolisms of larger $(11.11 \pm 0.32 \mathrm{~g})$ and smaller $(5.30 \pm 0.06 \mathrm{~g})$ individuals in the same batch of white shrimp cultured in the same environment through high-throughput sequencing of 16S rRNA gene. Our results showed that there was no significant difference in the composition of intestinal microbiota between larger and smaller white shrimps. However, Shewanella algae and Neptunomonas sp. were significantly enriched in the larger group, while Delftia sp., Hydrogenophaga sp., Pseudomonas sp., Synechococcus sp., Methylibium sp., Acidovorax sp., Limnohabitans sp., Burkholderia sp., Candidatus Koribacter sp., and Vogesella sp. were enriched in the smaller group. Moreover, the intestinal microbiota might promote the energy metabolism and growth of white shrimps through regulating their metabolic characteristics and switching their metabolisms from material synthesis to energy metabolisms.
\end{abstract}

Keywords: gut, microbiota structure, growth promoting probiotics, aquaculture, high-throughput sequencing

\section{Introduction}

Shrimp is a good protein source with low-fat content, and it is the most popular seafood product around the world. According to the statistics of the Food and Agriculture Organization of the United Nations (FAO), crustacean culture productions have expanded from 5478.8 thousand tonnes in 2010 to 9386.5 thousand tonnes in 2018 , with over $10 \%$ of annualized growth (FAO, 2020). The crustacean production is dominated by white shrimp (Litopenaeus vannamei), which has reached 4966.2 thousand tonnes and contributes to 52.9\% of the crustacean production in 2018 (FAO, 2020). Therefore, shrimp aquaculture plays a significant role in the human nutrition supply and world economy, and exploration of low feed consumption, high efficiency, low pollution and ecological white shrimp aquaculture technology has been an important topic in aquaculture.

Trillions of microorganisms inhabit the metazoan intestinal tract (termed intestinal microbiota), and they play an important role in host nutrition, development, growth, and health (Nieuwdorp et al., 2014; Magnúsdóttir et al., 2015; Yan et al., 2016; Miyamoto et al., 2019; Butt and Volkoff, 2019). For instance, by colonizing conventional specific pathogen-free intestinal microbiota into sexually mature germ-free mice and antibiotic treatment of conventional mice, as well as supplementation of antibiotic-treated mice with short-chain fatty acids (SCFAs), Yan et al. (2016) demonstrate that intestinal microbiota 
promotes the production of insulin-like growth factor 1 (IFG-1) in liver and adipose tissue through producing SCFAs and improve serum levels of IFG-1, which causes an increase in bone formation and growth. Storelli et al. (2011) found that Drosophila microbiota promotes larval growth upon nutrient scarcity, and Lactobacillus plantarum, a commensal bacterium of the Drosophila intestine, is sufficient on its own to recapitulate the natural microbiota growth-promoting effect by modulating hormonal signals through TOR-dependent nutrient sensing. Zheng et al. (2017) report that intestinal microbiota promotes weight gain of both whole body and the gut in individual honey bees likely through mediating changes in host vitellogenin, insulin signaling, and gustatory response.

The role of intestinal microbiota in the growth and health of shrimps has also been widely investigated (Xiong et al., 2015, 2017; Anuta et al., 2016; Holt et al., 2020). For instances, Xiong et al. (2017) evaluated the composition and ecological processes of the intestinal bacterial communities in cohabitating retarded, overgrown, and normal white shrimps from identically managed ponds, and they found that intestinal bacterial community structures were distinct among the shrimp categories. Moreover, they found that changes in the intestinal microbiota were positively related to digestive activities, which subsequently affected shrimp growth rate (Xiong et al., 2017). Huang et al. (2020) reported that white shrimp intestinal microbiota may partly be derived from large particle of bioflocs, and these bacteria driven by large particles may play an important role in promoting shrimp growth.

Although the intestinal bacterial communities in cohabitating retarded, overgrown, and normal white shrimps were reported there were significant differences (Xiong et al., 2017), how these bacteria affect the growth of white shrimp remains unclear. Considering the intestinal microbiota is widely involved in the host's metabolic regulation through its own metabolic process, we speculated that there were significant differences in the metabolic processes of intestinal microbiota between fast-growing and slow-growing individuals in the same batch of white shrimp. To test the speculation, in this study, we compared the intestinal microbiota compositions and metabolic characteristics of fastgrowing and slow-growing individuals in the same batch of white shrimp cultured in the same environment through high-throughput sequencing of 16S rRNA gene.

\section{Materials and Methods}

\section{Sample collection}

The experiment was conducted in the Qingjiang Base of the Zhejiang Institute of Marine Aquaculture. The seawater was taken from the Leqing Bay of East China Sea (salinity: $18.5 \pm 0.6 \%$ ). Mariculture seawater with salinity of $24-26 \%$ and $\mathrm{pH}$ of $8.2 \pm 0.3$ was obtained through secondary sand filtration in the impoundment pond. In the experiment, the larvae of the same pair of male and female white shrimps were cultured to approximately $1.0 \mathrm{~cm}$, then 10000 of them were put into an indoor culture cement pond with $5 \times 8 \times 1.5 \mathrm{~m}$ for breeding. The water was changed $1 / 3$ of total volume every 4 days, and the residual bait, shell, and dead prawns were cleaned. The larvae were bred for 130 days. During the experiment period, the larvae were fed with commercial prawn formula feed (Zhengda, Binzhou, China) at 6:00, 14:00, and 22:00. The daily feed weight was $15 \%$ of the prawn body weight. The pond was aerated continuously during the experiment period. The lighting cycle was natural light. The water temperature was $26 \pm 0.5^{\circ} \mathrm{C}$. 
At the end of the experiment, the white shrimps were divided into two groups according to their body length and body weight: larger (L) and smaller (S) groups. Each 10 white shrimps of $\mathrm{L}$ and $\mathrm{S}$ groups were randomly collected. After measuring the body length, body weight, and body width, the intestinal tract of each sample was dissected under sterile condition and put into a $2 \mathrm{ml}$ sterile centrifuge tube for subsequent extraction of total genomic DNA of intestinal microbiota. The hepatopancreas of the samples were also collected and stored at $-80{ }^{\circ} \mathrm{C}$ for determination of the activities of amylase and lipase. In addition, each two white shrimps of L and S groups were randomly collected, and their intestines were dissected and stored in $4 \%$ paraformaldehyde universal tissue fixative (Biosharp, Hefei, China) for analysis of intestinal tissue section.

\section{Determination of amylase and lipase activity of hepatopanceas}

The $\alpha$-amylase and lipase activity of hepatopancreas were determined using an $\alpha$ amylase ( $\alpha$-AMS) assay kit (Nanjing Jiancheng Bioengineering Institute, Nanjing, China) and a lipase (LPS) assay kit (Nanjing Jiancheng Bioengineering Institute, Nanjing, China, China), respectively.

\section{Analysis of intestinal tissue section}

The fixed intestinal tissues of prawns were paraffin embedded and sectioned, and the sections were stained with hematoxylin and eosin according to the method described by Fischer et al. (2008a,b,c).

\section{DNA extraction, PCR amplification and high-throughput sequencing}

Fecal microbial DNA was extracted using a PowerSoil DNA isolation kit (QIAGEN, Germany). Then the V4-V5 hypervariable region of the prokaryotic 16S rRNA gene was amplified using the universal primers $515 \mathrm{~F}$ and $909 \mathrm{R}$ with a 12-nt sample-specific barcode sequence included at the 5'-end of the $515 \mathrm{~F}$ primer to distinguish samples (Ni et al., 2019, 2021). Polymerase chain reaction (PCR) was performed in duplicate with a $25-\mu \mathrm{L}$ reaction mix containing $1 \times$ PCR buffer, $0.25 \mathrm{U}$ of Taq polymerase (Transgen, China), $0.2 \mathrm{mM}$ of each deoxynucleoside triphosphate (Transgen, China), $10 \mu \mathrm{M}$ of each primer (Sangon Biotech, China) and 10 ng microbial genomic DNA (Xiang et al., 2018). The thermal cycling procedure consisted at $94{ }^{\circ} \mathrm{C}$ for $10 \mathrm{~min}$, followed by 30 cycles of $94{ }^{\circ} \mathrm{C}$ for $30 \mathrm{~s}, 56{ }^{\circ} \mathrm{C}$ for $30 \mathrm{~s}$ and $72{ }^{\circ} \mathrm{C}$ for $30 \mathrm{~s}$, and finally $72{ }^{\circ} \mathrm{C}$ for $10 \mathrm{~min}$ (Xiang et al., 2018). Then the two PCR products from the same sample were mixed together and purified using a SanPrep DNA gel extraction kit (Sangon Biotech, China). All amplicons were pooled together with an equal molar amount from each sample and sequenced using an Illumina HiSeq system at Guangdong Meilikang Bio-Science, Ltd., China (Xiang et al., 2018).

Raw reads were merged using FLASH 1.2.8 (Magoc and Salzberg, 2011) and processed using QIIME pipeline 1.9.0 (Caporaso et al., 2010) as previously described (Xiang et al., 2018). Briefly, merged sequences were removed low-quality sequences and chimera sequences using QIIME pipeline 1.9.0 and UCHIME software (Edgar et al., 2011). Then, the high-quality sequences were clustered unto operational taxonomic units (OTUs) at 97\% identity using UPARSE software (Edgar, 2013). Subsequently, all samples were randomly resampled to obtain the same number of sequences using the single_rarefaction.py command of QIIME pipeline 1.9.0. Taxonomy of each OTU was assigned using the RDP classifier (Wang et al., 2007) with gg_13_8_otus database. 
Metabolic characteristics of the intestinal microbiota were predicted using the PICRUSt software based on the intestinal microbiota compositions (Langille et al., 2013).

\section{Data analysis}

Data were presented as the mean \pm standard error. Principal coordinate analysis (PCoA) was conducted using the QIIME pipeline 1.9.0. Nonparametric multivariate analysis of variance (PERMANOVA) (Anderson, 2001) was conducted using the vegan package (Dixon, 2003) of R 4.0.4 (R Core Team, 2013). Student's t test was used to compare the differences between larger and smaller prawn samples. Linear discriminant analysis effect size (LEfSe) (Segata et al., 2011) was analyzed on the Galaxy platform (http://huttenhower.sph.harvard.edu/galaxy/). Heatmap profiles of OUT compositions and KEGG ontologies were drown using pheatmap package of R 4.0.4.

\section{Results}

\section{Differences in the size, the length of hindgut villi, and a-amylase and lipase activity of} hepatopancreas between larger and smaller white shrimps

As expected, the body weight, body length, and body width of the L group were significantly higher than those of the $\mathrm{S}$ group (Student's t-test, $\mathrm{p}<0.001$; Fig. $1 \mathrm{~A}-1 \mathrm{C}$ ). The body weights of the $\mathrm{L}$ and $\mathrm{S}$ groups were $11.11 \pm 0.32 \mathrm{~g}$, and $5.30 \pm 0.06 \mathrm{~g} \mathrm{(Fig.} \mathrm{1A).}$ The body lengths of the $\mathrm{L}$ and $\mathrm{S}$ groups were $11.54 \pm 0.16 \mathrm{~cm}$, and $9.01 \pm 0.07 \mathrm{~cm}$ (Fig. 1B). However, results of intestinal tissue sections showed that the lengths of hindgut villi between the L and S groups was no significant difference (Student's t-test, $\mathrm{p}>0.05$; Fig. $1 D-1 F$ ). Moreover, $\alpha$-amylase and lipase activities of hepatopancreas were also no significant difference between the L and S groups (Student's t-test, p > 0.05; Fig. 2).

(A)

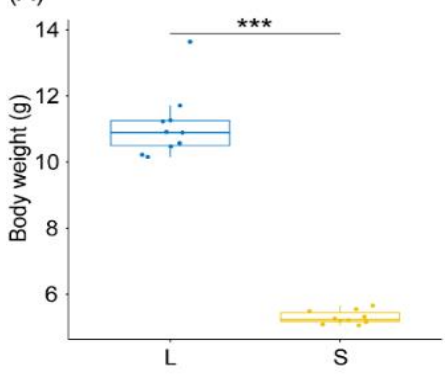

(D)

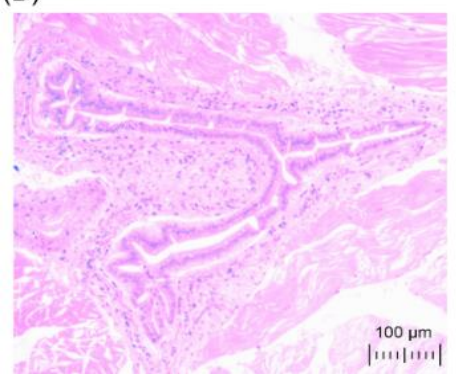

(B)

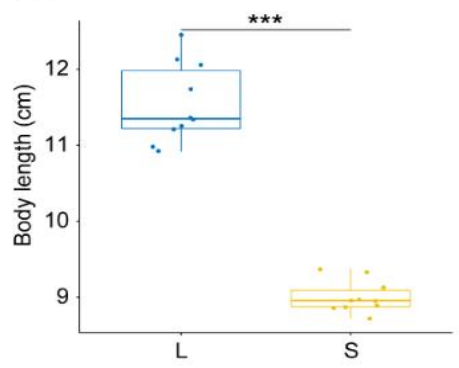

$(\mathrm{E})$

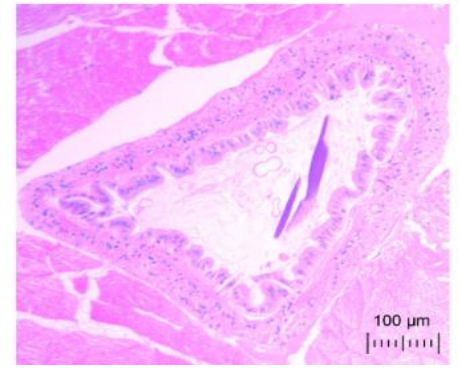

(C)

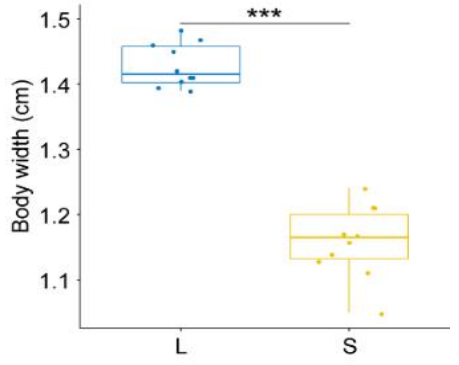

(F)

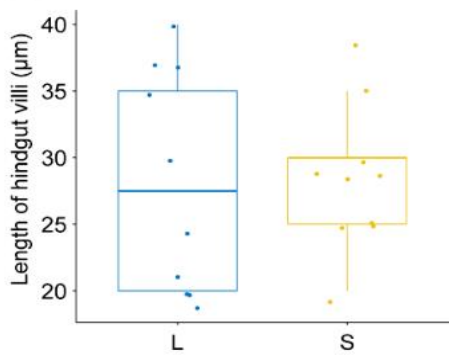

Figure 1. Differences in the size and the length of hindgut villi of white shrimps. L, larger group; S, smaller group. (A), body weight; (B), body length; $(C)$, body width; (D), hindgut section of larger group; $(E)$, hindgut section of smaller group; $(F)$, length of hindgut villi. ***, $p<0.001$ 
(A)

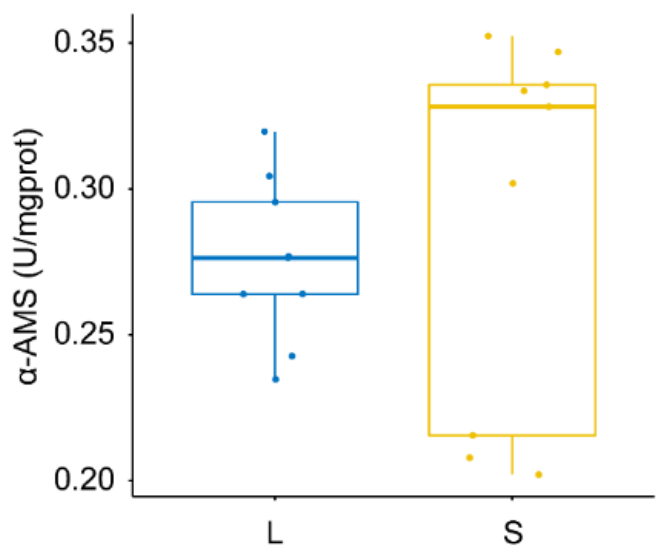

(B)

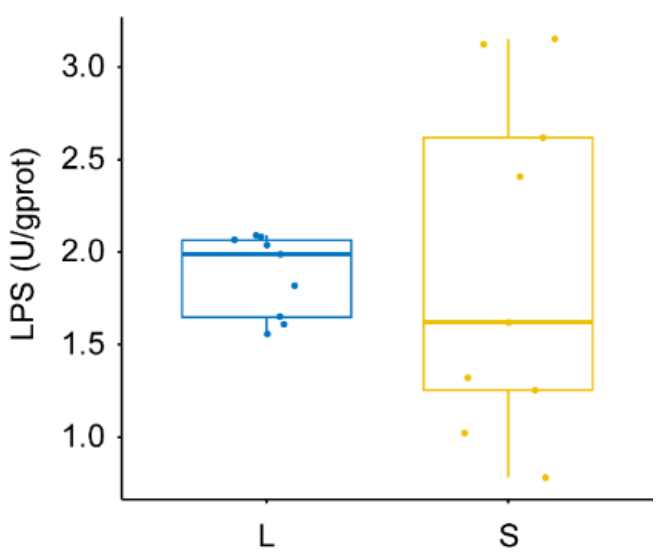

Figure 2. $\alpha$-Amylase and lipase activities of hepatopancreas between larger and smaller white shrimps. L, larger group; $S$, smaller group; $\alpha$-AMS, $\alpha$-amylase; LPS, lipase

\section{Composition differences of intestinal microbiota between larger and smaller white shrimps}

A total of 1,336,738 high-quality sequences were obtained from the 20 intestinal samples. Finally, 26,747 high-quality sequences were randomly selected from each sample for further analysis. PCoA with PERMANOVA showed that there was no significant difference in the OTU compositions of intestinal microbiota between the $\mathrm{L}$ and $\mathrm{S}$ groups (PERMANOVA, $\mathrm{F}=1.002, \mathrm{p}=0.408 ;$ Fig. 3A). Except for a few OTUs that could not be classified into any phylum, other OTUs were classified into 60 phyla (3 Archaea phyla and 57 Bacteria phyla), in which Proteobacteria, Bacteroidetes, Tenericutes, Acidobacteria, Actinobacteria, Chloroflexi, Cyanobacteria, Firmicutes, Fusobacteria, Nitrospirae, and Planctomycetes dominated the intestinal microbiota (Fig. 3B). Except the relative abundance of Tenericutes in the L group was significantly higher than that in the $S$ group, there was no significant difference in other dominant phyla between the L and S groups (White's non-parametric t-test, $\mathrm{p}>0.05 ;$ Fig. $3 \mathrm{C}$ ). It was worth noting that Proteobacteria has an absolute dominance in these intestinal microbiota, and its relative abundance was over $70 \%$ in all samples, and reached to 98.20\% in the sample L1 (Fig. $3 B$ and $3 C$ ). This was mainly due to the high relative abundance of Vibrio belonging to Proteobacteria in these intestinal microbiota, with a relative abundance of $68.95 \pm 0.04 \%$. However, there was no significant difference in the relative abundance of Vibrio between the $\mathrm{L}$ and $\mathrm{S}$ groups (Student's t-test, $\mathrm{t}=0.16$, $\mathrm{p}=0.87$; Fig. 3D).

To confirm that there is no significant difference in the intestinal microbiota between the L and S groups, we further analyzed the dominant OTUs in more detail. Our results showed that Shewanella algae and Neptunomonas sp. were significantly enriched in the L group, while Delftia sp., Hydrogenophaga sp., Pseudomonas sp., Synechococcus sp., Methylibium sp., Acidovorax sp., Limnohabitans sp., Burkholderia sp., Candidatus Koribacter sp., and Vogesella sp. were enriched in the S group (LEfSe, $\log _{10}$ LDA score $>2$; Fig. $4 A$ and $4 B$ ). Heatmap profile showed a similar result (Fig. 4C). 
(A)

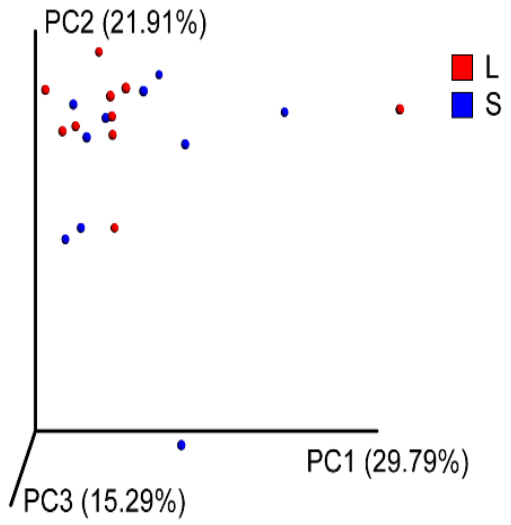

(C)

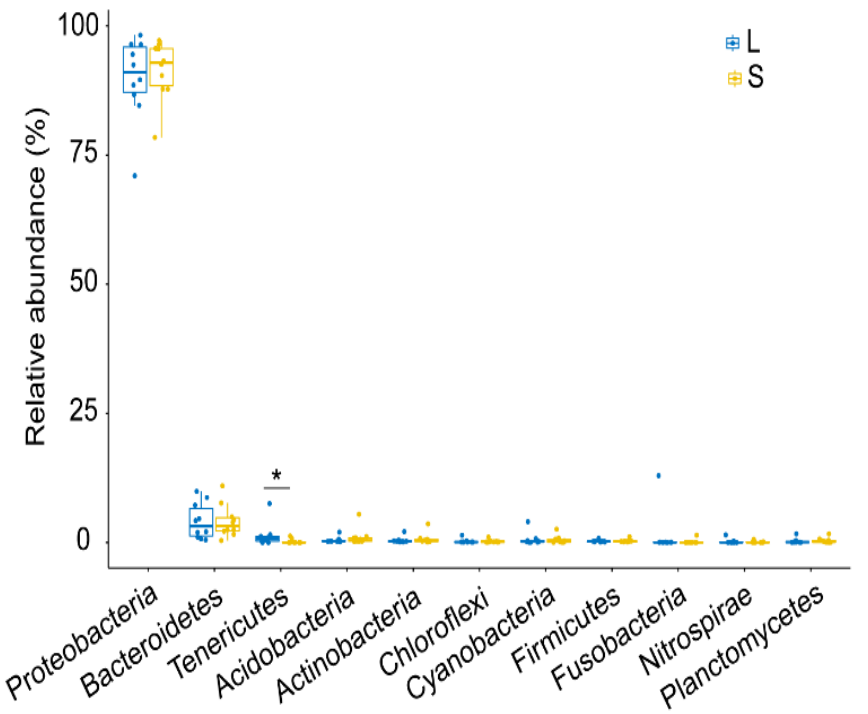

(B)

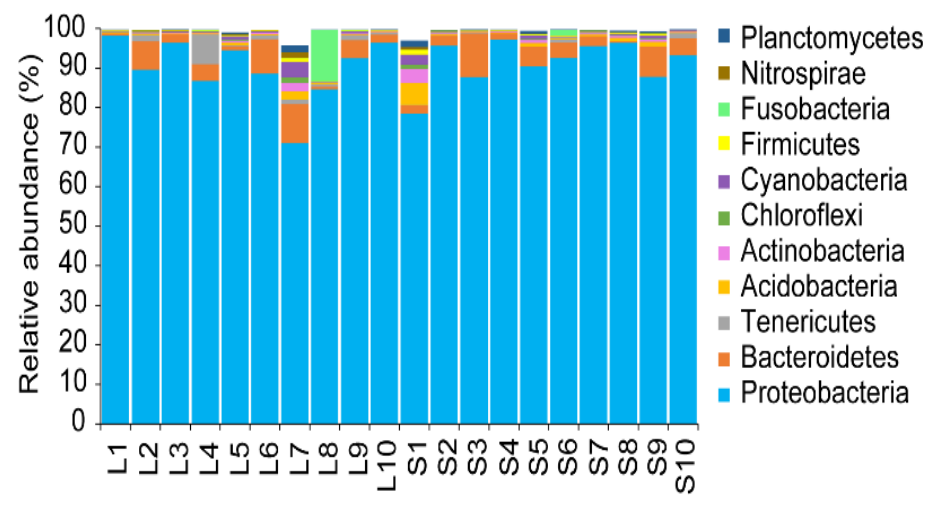

(D)

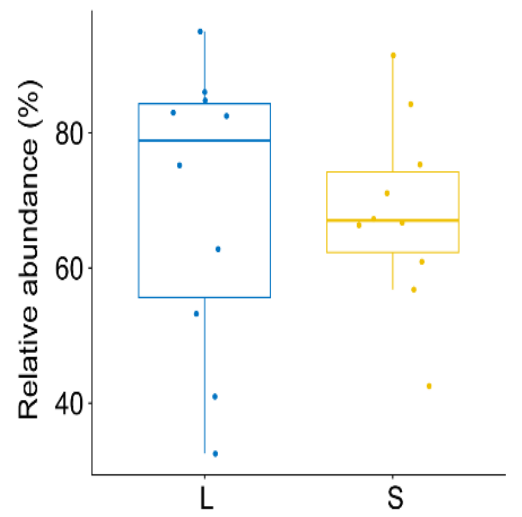

Figure 3. Intestinal microbiota compositions of larger and smaller white shrimps. (A), PCoA profile; $(B)$, relative abundances of dominant phyla; $(C)$, boxplots showed difference of dominant phyla; $(D)$, boxplot showed the relative abundance of Vibrio. L, larger group; $S$, smaller group. *, $P<0.05$

\section{Potential metabolic differences of intestinal microbiota between larger and smaller prawn samples}

To analyze the metabolic characteristics of intestinal microbiota of the $\mathrm{L}$ and $\mathrm{S}$ white shrimps, metabolic characteristics of the intestinal microbiota were predicted using the PICRUSt software based on the intestinal microbiota compositions. There were significant differences in 132 KEGG orthologies (KOs) between the L and S groups, in which the enhanced KOs in the L group were mainly involved in energy metabolisms and hydrolases, while the enhanced KOs in the S group were mainly involved in the cell structure synthesis, such as outer membrane protein, periplasmic protein, ribonucleotide synthase, and large subunit ribosomal protein (Fig. 5). 
(A)

$\square \mathrm{L} \square \mathrm{S}$

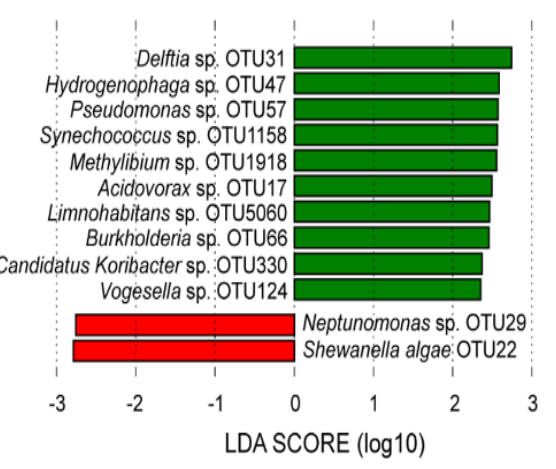

(B)

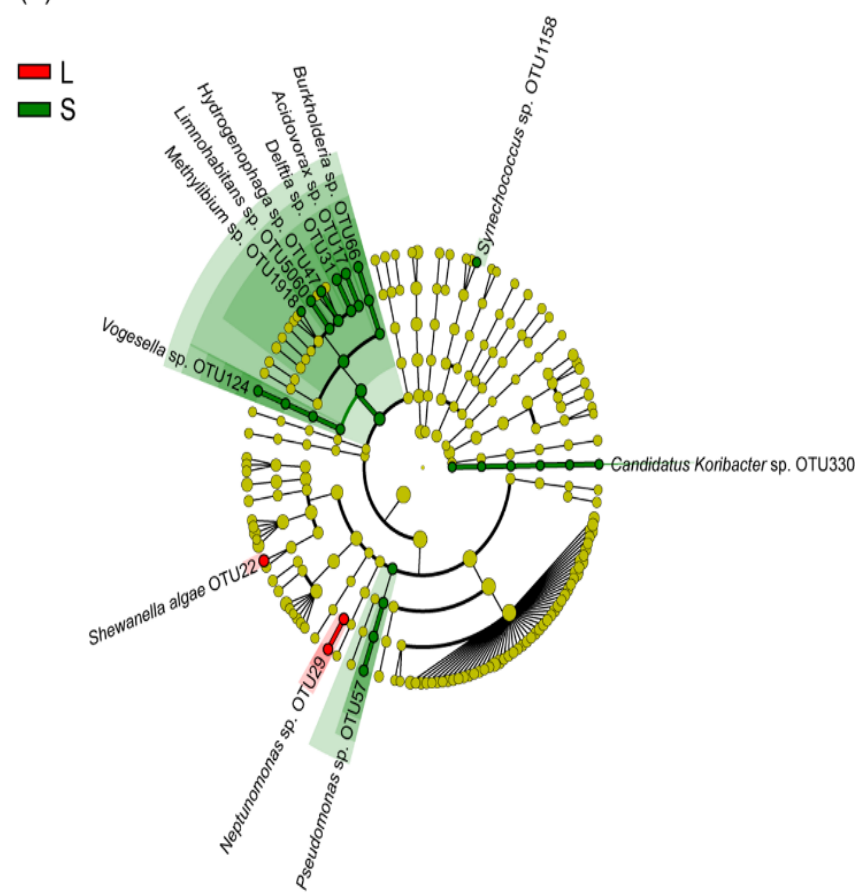

(C)

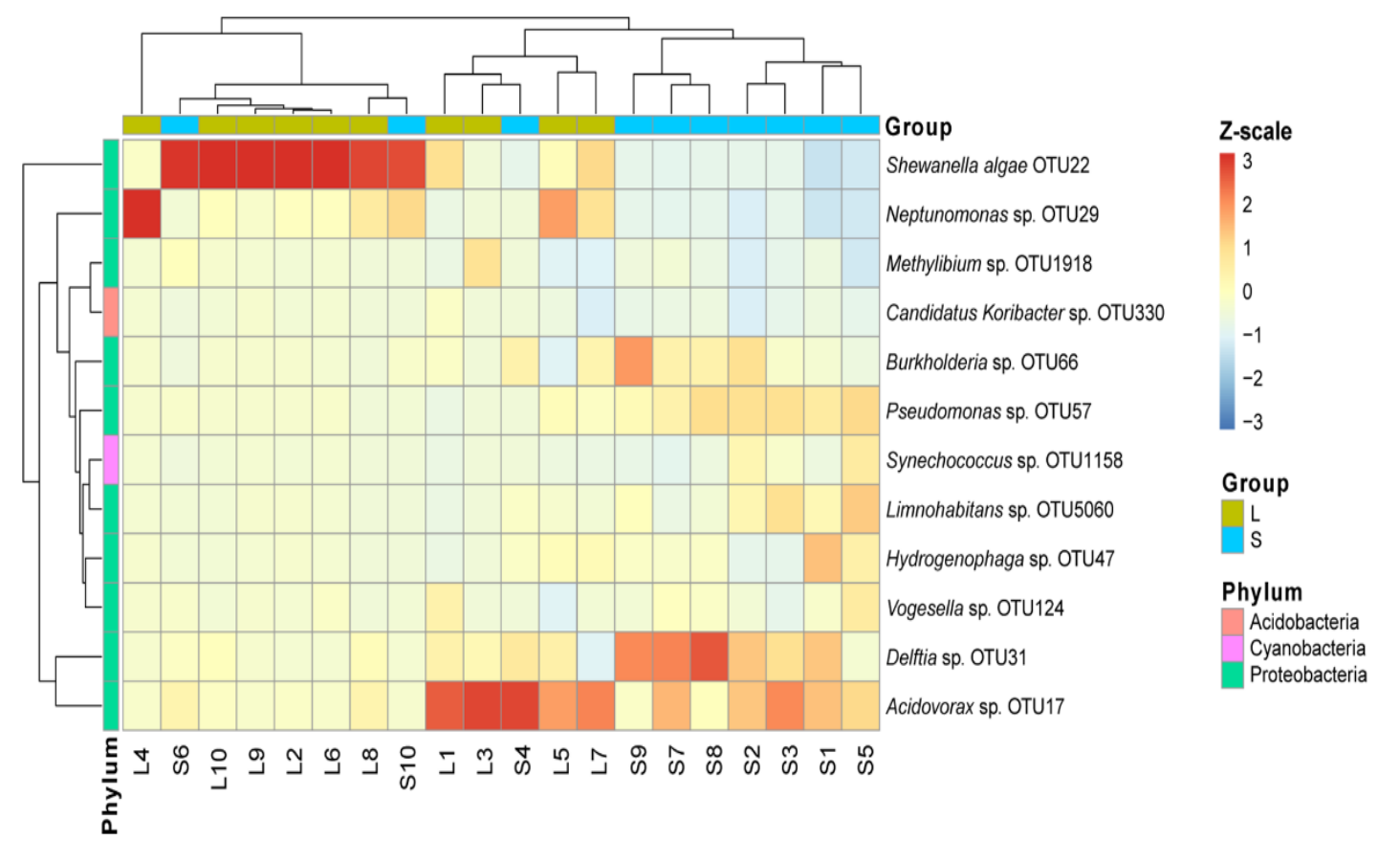

Figure 4. Different OTUs of intestinal microbiota between larger and smaller white shrimps. L, larger group; $S$, smaller group 


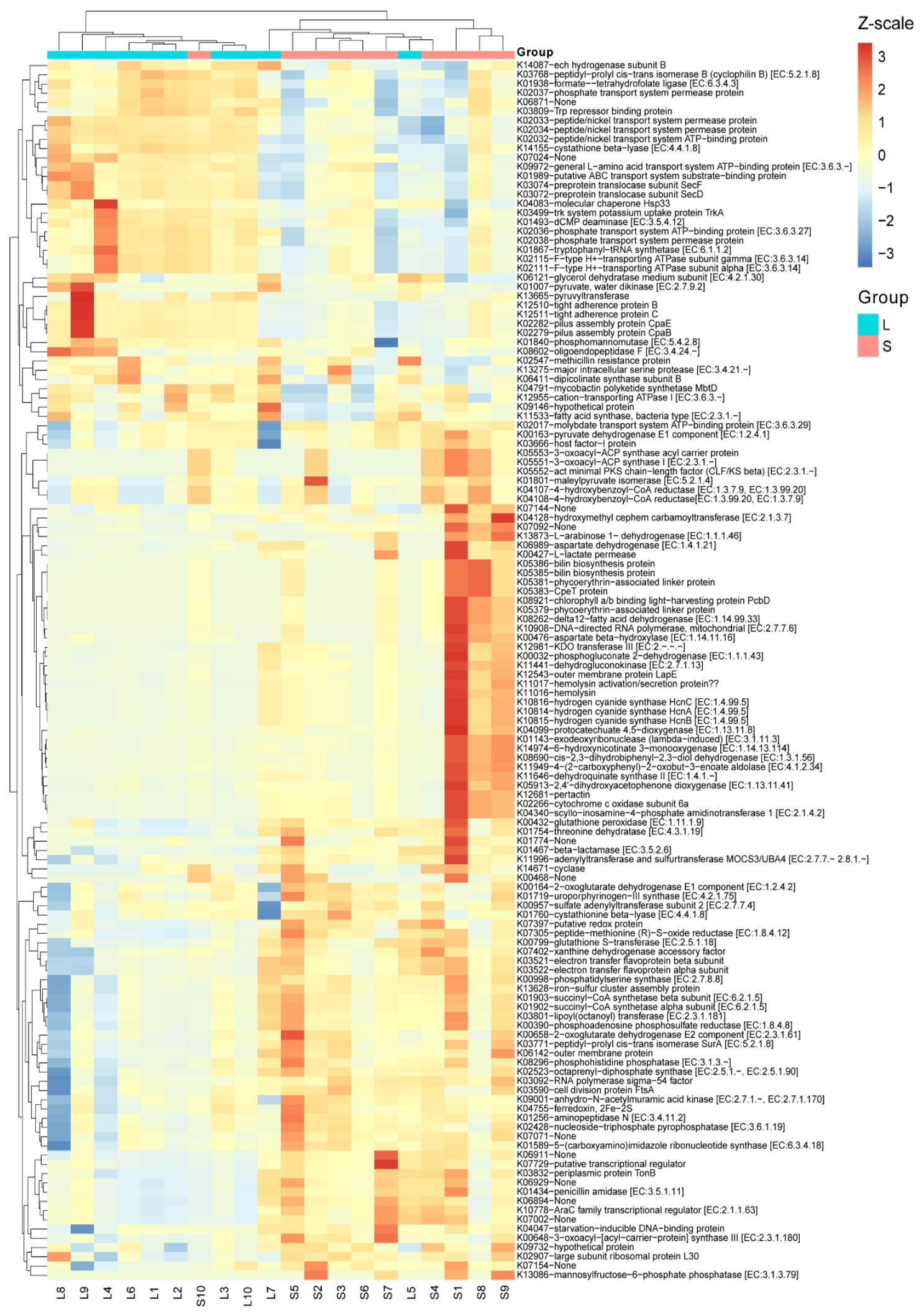

Figure 5. Heatmap profile shows significantly different KEGG ontologies between larger and smaller white shrimps. L, larger group; $S$, smaller group. 4-HBCR, 4-hydroxybenzoyl-CoA reductase; PPIB, peptidyl-prolyl cis-trans isomerase B; 3-Oxsm, 3-oxoacyl-ACP synthase; OPDS, octaprenyl-diphosphate synthase; ANMK, anhydro- $N$-acetylmuramic acid kinase; $C M T$, cysteine methyltransferase; RPAR, regulatory protein of adaptative response 


\section{Discussion}

Intestinal microbiota plays an important role in various physiological processes of host, such as growth, metabolism, immunity, and health through participating in the metabolism processes of host (Nieuwdorp et al., 2014; Magnúsdóttir et al., 2015; Yan et al., 2016; Wang et al., 2016; Oliphant and Allen Vercoe, 2019; Miyamoto et al., 2019; Butt and Volkoff, 2019; Ni et al., 2021). Although intestinal microbiota is closely related to host energy metabolism and obesity (Tremaroli and Bäckhed, 2012; Miyamoto et al., 2019), and it has been reported that there were significantly differences in intestinal microbiota between cohabitating retarded, overgrown, and normal shrimps (Xiong et al., 2017), our results did not show a significant difference in the whole intestinal microbiota compositions between the L and S groups of white shrimps (Fig. 3). Except for the significant differences in body length, body weight, and body width caused by sampling selection, the intestinal tissue structure and the $\alpha$-amylase and lipase activities of hepatopancreas were no significant difference between the larger and smaller white shrimps (Figs. 1 and 2). These results indicated that the growth of white shrimp was not directly affected by the structure changes of intestinal microbiota. However, we still detected significantly different bacteria of intestinal microbiota between the $\mathrm{S}$ and $\mathrm{L}$ groups (Fig. 4).

Shewanella and Neptunomonas are two bacterial genera which are widely distributed in marine and participate in energy metabolism and material circulation (Dubiel et al., 2002; Beleneva et al., 2009; Bargiela et al., 2015; Light et al., 2019). Although there are a few reports about Shewanella pathogens (Beleneva et al., 2009), there is no Shewanella pathogen reported in shrimps. Neptunomonas sp. 0536 was reported as probiotic to suppress naturally occurring vibrios in the culture environment of healthy mussel larvae (Kesarcodi-Watson et al., 2010). The enriched bacteria in the $S$ group, such as those in Delftia, Hydrogenophaga, Synechococcus and Methylibium, were also widely reported as degrading bacteria of organic pollutants (Streger et al., 2002; Moore et al., 2002; Choi et al., 2003; Kane et al., 2007; Jørgensen et al., 2009; Zhang et al., 2010). These results implied that these significantly different bacteria might affect the growth of enriched in the $\mathrm{S}$ group through participating in different material or energy metabolisms.

Intestinal microbiota plays an important role in host material and energy metabolisms (Tremaroli and Bäckhed, 2012). However, due to different characteristics of energy and substance transfer, energy is easier to be shared between the host and the intestinal microbiota, while substances are more difficult to be shared between the host and the intestinal microbiota except those extracellular secondary metabolites. Moreover, intestinal microbiota utilizes intestinal substances to synthesize their cell structures, and compete with the host for nutrients, which may limit the host's access to nutrients. Our results showed that the functional genes enhanced in the L group were mainly involved in enzymes of energy metabolism and hydrolases, while those in the $\mathrm{S}$ group were mainly involved in cell structure synthesis. This might be an important reason for the growth difference between the L and S groups.

Compositions of intestinal microbiota is easily affected by external environmental factors (Xiong et al., 2015; Zhao et al., 2018; Fan et al., 2019; Zhang et al., 2021). Therefore, in this study, the group internal error of intestinal microbiota caused by the undetected factors covered the difference between the L and S groups, which leaded to no significant difference in the compositions of intestinal microbiota between the $\mathrm{L}$ and $\mathrm{S}$ groups was detected. These results showed that in the study of the relationship between 
intestinal microbiota and host, we should not only focus on the composition of intestinal microbiota, especially if the environmental variables are not well controlled.

\section{Conclusions}

Our results showed that there was no significant difference in the composition of intestinal microbiota between larger and smaller white shrimps. However, Shewanella algae and Neptunomonas sp. were significantly enriched in the L group, while Delftia sp., Hydrogenophaga sp., Pseudomonas sp., Synechococcus sp., Methylibium sp., Acidovorax sp., Limnohabitans sp., Burkholderia sp., Candidatus Koribacter sp., and Vogesella sp. were enriched in the $\mathrm{S}$ group. Moreover, the intestinal microbiota might promote the energy metabolism and growth of white shrimps through regulating their metabolic characteristics and switching their metabolisms from material synthesis to energy metabolisms. However, further study is needed on what factors cause the differences of intestinal microbiota metabolic characteristics between the $\mathrm{L}$ and $\mathrm{S}$ groups, and how to regulate the intestinal microbiota metabolic characteristics to promote the growth of white shrimps.

Acknowledgements. This work was supported by the National Key Research and Development Plan of China (grant No. 2020YFD0900801), the Major Science and Technology Project of New Agricultural Varieties Breeding in Zhejiang Province (grant No. 2021C02025-5) and the Agricultural New Variety Selection and Breeding Cooperation Group Project of Wenzhou City (grant No. 2019ZX002-01). We would like to thank anonymous technicians at Guangdong Meilikang Bio-Science Ltd., China for assistance with data re-analysis.

\section{REFERENCES}

[1] Anderson, M. J. (2001): A new method for non-parametric multivariate analysis of variance. - Austral Ecology 26: 32-46.

[2] Anuta, J. D., Buentello, A., Patnaik, S., Hume, M. E., Mustafa, A., Gatlin, D. M. III, Lawrence, A. L. (2016): Effects of dietary supplementation of a commercial prebiotic Previda ${ }^{\circledR}$ on survival, growth, immune responses and gut microbiota of Pacific white shrimp, Litopenaeus vannamei. - Aquaculture Nutrition 22(2): 410-418. doi:10.1111/anu.12257.

[3] Bargiela, R., Mapelli, F., Rojo, D., Chouaia, B., Tornés, J., Borin, S., Richter, M., Del Pozo, M. V., Cappello, S., Gertler, C., et al. (2015): Bacterial population and biodegradation potential in chronically crude oil-contaminated marine sediments are strongly linked to temperature. - Scientific Reports 5: 11651.

[4] Beleneva, I. A., Magarlamov, T. Y., Eliseikina, M. G., Zhukova, N. V. (2009): Biochemical and pathogenic properties of the natural isolate of Shewanella algae from Peter the Great Bay, Sea of Japan. - Journal of Invertebrate Pathology 102: 250-255. doi:10.1016/j.jip.2009.09.001.

[5] Butt, R. L., Volkoff, H. (2019): Gut microbiota and energy homeostasis in fish. - Frontiers in Endocrinology 10: 9. doi:10.3389/fendo.2019.00009.

[6] Caporaso, J. G., Kuczynski, J., Stombaugh, J., Bittinger, K., Bushman, F. D., Costello, E. K., et al. (2010): QIIME allows analysis of high-throughput community sequencing data. - Nature Methods 7: 335-336. doi:10.1038/nmeth.f.303.

[7] Choi, M. H., Lee, H. J., Rho, J. K., Yoon, S. C., Nam, J. D., Lim, D., Lenz, R. W. (2003): Biosynthesis and local sequence specific degradation of poly (3-hydroxyvalerate-c o-4hydroxybutyrate) in Hydrogenophage pseudoflava. - Biomacromolecules 4: 38-45. 
[8] Dixon, P. (2003): VEGAN, a package of R functions for community ecology. - Journal of Vegetation Science 14: 927-930. doi:10.1111/j.1654-1103.2003.tb02228.x.

[9] Dubiel, M., Hsu, C. H., Chien, C. C., Mansfeld, F., Newman, D. K. (2002): Microbial iron respiration can protect steel from corrosion. - Applied and Environmental Microbiology 68(3): 1440-1445. doi:10.1128/AEM.68.3.1440-1445.2002.

[10] Edgar, R. C. (2013): UPARSE: Highly accurate OTU sequences from microbial amplicon reads. - Nature Methods 10: 996-998. doi:10.1038/nmeth.2604.

[11] Edgar, R. C., Haas, B. J., Clemente, J. C., Quince, C., Knight, R. (2011): UCHIME improves sensitivity and speed of chimera detection. - Bioinformatics 27: 2194-2200. doi:10.1093/bioinformatics/btr381.

[12] Fan, J., Chen, L., Mai, G., Zhang, H., Yang, J., Deng, D., Ma, Y. (2019): Dynamics of the gut microbiota in developmental stages of Litopenaeus vannamei reveal its association with body weight. - Scientific Reports 9: 734. doi:10.1038/s41598-018-37042-3.

[13] FAO. (2020): The State of World Fisheries and Aquaculture 2020. Sustainability in action. - The Organization of Food and Agriculture of the United Nations, Rome. http://www.fao.org/3/ca9229en/ca9229en.

[14] Fischer, A. H., Jacobson, K. A., Rose, J., Zeller, R. (2008a): Paraffin embedding tissue samples for sectioning. - Cold Spring Harbor Protocols 3(5): prot4989. doi:10.1101/pdb.prot4989.

[15] Fischer, A. H., Jacobson, K. A., Rose, J., Zeller, R. (2008b): Cutting sections of paraffinembedded tissues. - Cold Spring Harbor Protocols 3(5): prot4987. doi:10.1101/pdb.prot4987.

[16] Fischer, A. H., Jacobson, K. A., Rose, J., Zeller, R. (2008c): Hematoxylin and eosin staining of tissue and cell sections. - Cold Spring Harbor Protocols 3(5): prot4986. doi:10.1101/pdb.prot4986.

[17] Holt, C. C., Bass, D., Stentiford, G. D., van der Giezen, M. (2020): Understanding the role of the shrimp gut microbiome in health and disease. - Journal of Invertebrate Pathology, In press. doi:10.1016/j.jip.2020.107387.

[18] Huang, L., Guo, H., Chen, C., Huang, X., Chen, W., Bao, F., Liu, W., Wang, S., Zhang, D. (2020): The bacteria from large-sized bioflocs are more associated with the shrimp gut microbiota in culture system. - Aquaculture 523: 735159. doi:10.1016/j.aquaculture.2020.735159.

[19] Jørgensen, N. O. G., Brandt, K. K., Nybroe, O., Hansen, M. (2009): Delftia lacustris sp. nov., a peptidoglycan-degrading bacterium from fresh water, and emended description of Delftia tsuruhatensis as a peptidoglycan-degrading bacterium. - International Journal of Systematic and Evolutionary Microbiology 59: 2195-2199. doi:10.1099/ijs.0.008375-0.

[20] Kane, S. R., Chakicherla, A. Y., Chain, P. S. G., Schmidt, R., Shin, M. W., Legler, T. S., Scow, K. M., Larimer, F. W., Lucas, S. M., Richardson, P. M., Hristova, K. R. (2007): Whole-genome analysis of the methyl tert-butyl ether-degrading beta-proteobacterium Methylibium petroleiphilum PM1. - Journal of Bacteriology 189(5): 1931-1945.

[21] Kesarcodi-Watson, A., Kaspar, H., Lategan, J., Gibson, L. (2010): Alteromonas macleodii 0444 and Neptunomonas sp. 0536, two novel probiotics for hatchery-reared Greenshell ${ }^{\mathrm{TM}}$ mussel larvae, Perna canaliculus. - Aquaculture 309(1-4): 49-55.

10.1016/j.aquaculture.2010.09.019.

[22] Langille, M. G. I., Zaneveld, J., Caporaso, J. G., McDonald, D., Knights, D., Reyes, J. A., Clemente, J. C., Burkepile, D. E., Thurber, R. L. V., Knight, R., Beiko, R. G., Huttenhower, C. (2013): Predictive functional profiling of microbial communities using 16S rRNA marker gene sequences. - Nature Biotechnology 31(9): 814-921. doi:10.1038/nbt.2676.

[23] Light, S. H., Méheust, R., Ferrell, J. L., Cho, J., Deng, D., Agostoni, M., Iavarone, A. T., Banfield, J. F., D’Orazio, S. E. F., Portnoy, D. A. (2019): Extracellular electron transfer powers flavinylated extracellular reductases in Gram-positive bacteria. - Proceeding of the National Academy of Sciences of the United States of America 116(52): 26892-26899. doi:10.1073/pnas.1915678116. 
[24] Magnúsdóttir, S., Ravcheev, D., de Crécy-Lagard, V., Thiele, I. (2015): Systematic genome assessment of B-vitamin biosynthesis suggests co-operation among gut microbes. Frontiers in Genetics 6: 148. doi:10.3389/fgene.2015.00148.

[25] Magoc, T., Salzberg, S. L. (2011): FLASH: Fast length adjustment of short reads to improve genome assemblies. - Bioinformatics 27: 2957-2963. doi:10.1093/bioinformatics/btr507.

[26] Miyamoto, J., Igarashi, M., Watanabe, K., Karaki, S.-I., Mukouyama, H., Kishino, S., Li, X., Ichimura, A., Irie, J., Sugimoto, Y., Mizutani, T., Sugawara, T., Miki, T., Ogawa, J., Drucker, D. J., Arita, M., Itoh, H., Kimura, I. (2019): Gut microbiota confers host resistance to obesity by metabolizing dietary polyunsaturated fatty acids. - Nature Communications 10: 4007. doi:10.1038/s41467-019-11978-0.

[27] Moore, L. R., Post, A. F., Rocap, G., Chisholm, S. W. (2002): Utilization of different nitrogen sources by the marine cyanobacteria Prochlorococcus and Synechococcus. Limnology and Oceanography 47(4): 989-996.

[28] Ni, J., Huang, R., Zhou, H., Xu, X., Li, Y., Cao, P., Zhong, K., Ge, M., Chen, X., Hou, B., Yu, M., Peng, B., Li, Q., Zhang, P., Gao, Y. (2019): Analysis of the relationship between the degree of dysbiosis in gut microbiota and prognosis at different stages of primary hepatocellular carcinoma. - Frontiers in Microbiology 10: 1458.

doi:10.3389/fmicb.2019.01458.

[29] Ni, J., Fu, C., Huang, R., Li, Z., Li, S., Cao, P., Zhong, K., Ge, M., Gao, Y. (2021): Metabolic syndrome cannot mask the changes of faecal microbiota compositions caused by primary hepatocellular carcinoma. - Letters in Applied Microbiology 73(1): 73-80. doi:10.1111/lam.13477.

[30] Nieuwdorp, M., Gilijamse, P. W., Pai, N., Kaplan, L. M. (2014): Role of the microbiome in energy regulation and metabolism. - Gastroenterology 146(6): 1525-1533.

[31] Oliphant, K., Allen-Vercoe, E. (2019): Macronutrient metabolism by the human gut microbiome: major fermentation byproducts and their impact on host health. - Microbiome 7: 91. doi:10.1186/s40168-019-0704-8.

[32] R Core Team. (2013): R: A Language and Environment for Statistical Computing. - R Foundation for Statistical Computing, Vienna, Austria. URL http://www.R-project.org/.

[33] Segata, N., Izard, J., Waldron, L., Gevers, D., Miropolsky, L., Garrett, W. S., Huttenhower, C. (2011): Metagenomic biomarker discovery and explanation. - Genome Biology 12: R60. doi:10.1186/gb-2011-12-6-r60.

[34] Storelli, G., Defaye, A., Erkosar, B., Hols, P., Royet, J., Leulier, F. (2011): Lactobacillus plantarum promotes Drosophila systemic growth by modulating hormonal signals through TOR-dependent nutrient sensing. - Cell Metabomism 14: 403-414. doi:10.1016/j.cmet.2011.07.012.

[35] Streger, S. H., Vainberg, S., Dong, H., Hatzinger, P. B. (2002): Enhancing transport of Hydrogenophaga flava ENV735 for bioaugmentation of aquifers contaminated with methyl tert-butyl ether. - Applied and Environmental Microbiology 68(11): 5571-5579. doi:10.1128/AEM.68.11.5571-5579.2002.

[36] Tremaroli, V., Bäckhed, F. (2012): Functional interactions between the gut microbiota and host metabolism. - Nature 489: 242-249. doi:10.1038/nature11552.

[37] Wang, Q., Garrity, G. M., Tiedje, J. M., Cole, J. R. (2007): Naïve Bayesian classifier for rapid assignment of rRNA sequences into the new bacterial taxonomy. - Applied and Environmental Microbiology 73: 5261-5267. doi:10.1128/AEM.00062-07.

[38] Wang, Z., Koonen, D., Hofker, M., Fu, J. (2016): Gut microbiome and lipid metabolism: from associations to mechanisms. - Current Opinion in Lipidology 27(3): 216-224. doi:10.1097/MOL.0000000000000308.

[39] Xiang, J., He, T., Wang, P., Xie, M., Xiang, J., Ni, J. (2018): Opportunistic pathogens are abundant in the gut of cultured giant spiny frog (Paa spinosa). - Aquaculture Research 49: 2033-2041. doi:10.1111/are.13660. 
[40] Xiong, J., Wang, K., Wu, J., Qiuqian, L., Yang, K., Qian, Y., Zhang, D. (2015): Changes in intestinal bacterial communities are closely associated with shrimp disease severity. Applied Microbiology and Biotechnology 99: 6911-6919. doi:10.1007/s00253-015-6632$\mathrm{z}$.

[41] Xiong, J., Dai, W., Zhu, J., Liu, K., Dong, C., Qiu, Q. (2017): The underlying ecological processes of gut microbiota among cohabitating retarded, overgrown and normal shrimps. - Microbial Ecology 73: 988-999.

[42] Yan, J., Herzog, J. W., Tsang, K., Brennan, C. A., Bower, M. A., Garrett, W. S., Sartor, B. R., Aliprantis, A. O., Charles, J. F. (2016): Gut microbiota induce IGF-1 and promote bone formation and growth. - Proceeding of the National Academy of Sciences of the United States of America 113(47): E7554-E7563. doi.org/10.1073/pnas.1607235113.

[43] Zhang, L. L., He, D., Chen, J. M., Liu, Y. (2010): Biodegradation of 2-chloroaniline, 3chloroaniline, and 4-chloroaniline by a novel strain Delftia tsuruhatensis H1. - Journal of Hazardous Materials 179(1-3): 875-882. doi: 10.1016/j.jhazmat.2010.03.086.

[44] Zhang, X., Li, X., Lu, J., Qiu, Q., Chen, J., Xiong, J. (2021): Quantifying the importance of external and internal sources to the gut microbiota in juvenile and adult shrimp. Aquaculture 531: 735910. doi:10.1016/j.aquaculture.2020.735910.

[45] Zhao, Y., Duan, C., Zhang, X., Chen, H., Ren, H., Yin, Y., Ye, L. (2018): Insights into the gut microbiota of freshwater shrimp and its associations with the surrounding microbiota and environmental factors. - Journal of Microbiology and Biotechnology 28(6): 946-956. doi:10.4014/jmb.1710.09070.

[46] Zheng, H., Powell, J. E., Steele, M. I., Dietrich, C., Moran, N. A. (2017): Honeybee gut microbiota promotes host weight gain via bacterial metabolism and hormonal signaling. Proceedings of the National Academy of Sciences of the United States of America 114(18): 4775-4780. doi:10.1073/pnas.1701819114. 